\title{
The effects of an active-assisted stretching program on functional performance in elderly persons: A pilot study
}

\author{
Damian C Stanziano ${ }^{1,2}$ \\ Bernard A Roos 1,2,3,4 \\ Arlette C Perry' \\ Shenghan $\mathrm{Lai}^{5}$ \\ Joseph F Signorile ${ }^{1,3}$
}

'Department of Exercise and Sport Sciences, University of Miami, Coral Gables, FL, USA; ${ }^{2}$ Stein Gerontological Institute, Miami Jewish Home and Hospital, Miami, FL, USA; ${ }^{3}$ Geriatric Research, Education, and Clinical Center, Miami VA Healthcare System, Miami, FL, USA; ${ }^{4}$ Departments of Medicine and Neurology, University of Miami Miller School of Medicine, Miami, FL, USA; ${ }^{5}$ Departments of Pathology and Medicine, Johns Hopkins School of Medicine, Baltimore, MD, USA
Correspondence: Joseph F Signorile Department of Exercise and Sport Sciences, University of Miami, PO Box 248065, Coral Gables, FL 33124, USA

$\mathrm{Tel}+|\mathbf{3 0 5} 898| 457$

Fax +I 3055753365

Email jsignorile@miami.edu

\begin{abstract}
This study examined the impact of an eight-week active-assisted (AA) stretching program on functionality, mobility, power, and range of motion (ROM) in elderly residents of a residential retirement community. Seventeen volunteers ( 4 male, 13 female; $88.8 \pm 5.36$ years) were randomly assigned to an AA or control group. The AA group performed 10 different AA stretches targeting the major joints of the body twice weekly for eight weeks. Controls attended classes requiring limited physical activity. All participants were assessed using four flexibility and six functional tests, one week before and after the eight-week training period. A fully randomized repeated-measures ANCOVA with pretest scores as a covariate was used to detect differences between groups across time. The AA group demonstrated significant increases in ROM for most of the joints evaluated $(p<0.05)$ and significant increases in all performance measures $(p<0.05)$. Controls showed no improvements in functional or ROM measures $(\alpha=0.05$ ). Additionally, the AA group showed significantly better performance outcomes across the training period than controls. We conclude that our eight-week flexibility program effectively reduces age-related losses in ROM and improves functional performance in elderly persons with insufficient physical reserves to perform higher-intensity exercises.
\end{abstract}

Keywords: proprioceptive neuromuscular facilitation, activities of daily living, instrumental activities of daily living, functionality, exercise, quality of life

\section{Introduction}

Age-related declines in range of motion (ROM) are associated with decreases in mobility and activities of daily living (ADL) performance. ${ }^{1,2}$ In addition, studies with older persons have shown an association between improved flexibility and increases in ADL/instrumental ADL (IADL) performance and quality of life. ${ }^{3-5}$

This study uniquely relies on active-assisted (AA) stretching, a form of proprioceptive neuromuscular facilitation (PNF), as the sole intervention to improve flexibility and functional performance. In AA stretching, the target muscle is actively lengthened by the contraction of its antagonist. In this technique reciprocal inhibition facilitates the lengthening of the target muscle. The movement is then assisted by additional force application. PNF stretching has been shown to be more effective than static stretching at increasing ROM. ${ }^{6-8}$ Although an earlier study performed in our laboratory ${ }^{4}$ suggested that AA stretching would increase functional performance in older persons, no controlled study has documented its benefit in this population. The purpose of the randomized controlled study described in this report was to examine the impact of an AA flexibility program on ROM and functional performance variables in older persons living in a residential retirement community (RRC), serving both dependent and independent elderly persons. The study's results may provide useful information concerning the feasibility of using AA stretching to increase independence and mobility in frail older persons. 


\section{Methods}

\section{Participants}

Participants included 17 men and women $(88.7 \pm 5.4$ years old) residing in an RRC for an average of $2.6 \pm 1.6$ years. They were randomly assigned to the experimental group ( 1 man, 7 women; $90 \pm 4.5$ years old) or the control group ( 3 men, 6 women; $87.5 \pm 6.2$ years old). The groups were not found to be significantly different for age $(p=0.34)$ or body weight ( $p=0.87$ ). Potential participants were checked by their primary care physicians for disorders that would prevent their participation in a low-intensity, chair-based exercise study. Exclusion criteria were a) not living in an RRC, and b) inability to sit upright in a chair for thirty minutes due to akathisia or neurological or osteoporotic limitations. Once cleared by their physicians and before participation commenced, all participants signed an informed consent form approved by the University of Miami Subcommittee for the Use of Patients or Human Subjects and the Miami Jewish Home and Hospital Committee for the Use and Protection of Human Subjects.

\section{Procedures}

Figure 1 presents a randomized control trial chart for the present study. In advance of the eight-week training period, participants in the AA group were introduced to the concept of AA stretching and taught the proper technique for each exercise, including the contraction of the antagonist muscle group to facilitate the stretch of the targeted muscle group. They were also shown how to increase the stretch further by actively pulling on a rope (Flexmate, Promise Enterprises, Miami, FL) looped across the limb to be stretched or by pulling with a free hand on the limb to be stretched. All participants performed 10 repetitions of 10 different stretches, holding each repetition for about 4 to 5 seconds. A research staff member led the AA flexibility classes two days per week for approximately 30 minutes, in which participants were carefully monitored by a second staff member. Classes were held in an activity room in the lobby of the participants' residences. The 10 stretches used in the study were the back scratch (shoulder flexion/abduction); back arm press (shoulder hyperextension); standing thigh (hip hyperextension); side lunge (hip abduction); overhead back (shoulder hyperflexion); overhead side (lateral trunk flexion); cross chest (horizontal shoulder adduction); seated trunk twist (trunk rotation); seated hamstring (trunk/hip flexion); and seated calf (dorsiflexion). The control group participated in an arts and craft class which required limited physical

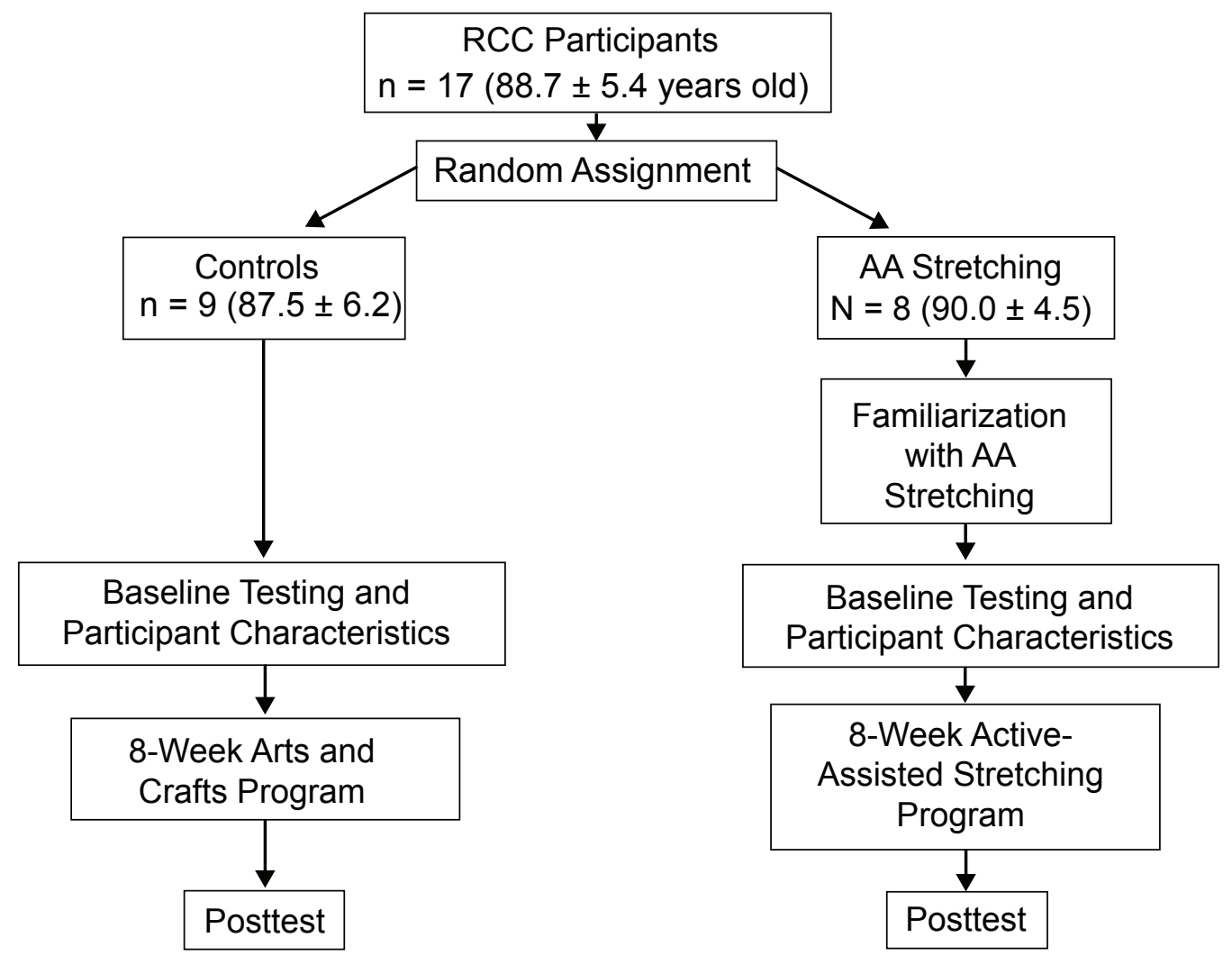

Figure I Randomized controlled trial flow chart. 
exertion during the eight-week period between testing sessions. Attendance was taken at each class session, and any participant missing more than three sessions was not included in the analysis. A total of four participants were not included in the analysis (3 AA; $1 \mathrm{C}$ ).

Testing was done one week before and after the training period. All tests were administered in a single session by the same researcher and were preceded by detailed instructions and practice trials. The selected tests are reliable, valid, and sensitive measures of functional performance in older persons. Tests were categorized as measures of flexibility, functional power, or mobility according to the outcome variable.

Flexibility was assessed by four tests: the back-scratch test (BS) $;{ }^{9}$ the modified chair sit-and-reach test (SR) ${ }^{9}$ the supine knee-extension test (KE); ${ }^{10}$ and the modified total body rotation test (BR). ${ }^{11}$ All tests were performed in accordance with testing protocols previously described in the literature, with the exception of the BR test. In the original BR test, the subject stood an arm's length from the wall and rotated away from the wall, reaching as far back as possible without moving the feet or bending the elbow. The reach was made by the arm on the side of the body not facing the wall, and the tester measured how far forward along the wall the participant's hand reached. In the current study, the test was modified so the subject rotated toward the wall, and the tester measured how far back the participant could reach without moving his or her feet. This modification increased safety, decreased subject trepidation, and reduced any basement effect that might exist with an older population.

The tests chosen to assess lower body strength and power were the 30 -second chair stand test (CS $)^{9}$ and modified ramp power test (MRPT), ${ }^{12}$ respectively. Upper body strength was assessed by the 30 -second arm curl (AC), ${ }^{9}$ and upper body, as well as core power, was evaluated by the gallon jug shelf test (GJST). ${ }^{13}$

The two tests used to assess mobility were the 50-foot gait speed test (GS) ${ }^{14}$ and the 8 -foot up-and-go test (UG). ${ }^{9}$ Both of these tests have been used extensively with older persons, and their validity and reliability have been confirmed in this population. ${ }^{9,12-14}$

In our hands, Cronbach's alpha scores for the BS and SR tests ranged from 0.927 to 0.983 , while the scores for the GJST, MRPT, and GS tests ranged from 0.939 to 0.987 when examined in samples of community-dwelling older persons aged 55-89 years. Reliability values for the other tests used in this study are not available; however, given the straightforward nature of the tests used, we assume that the reliability of these tests would be similar.

\section{Statistical analyses}

Pretest $\left(\mathrm{T}_{0}\right)$ and posttest $\left(\mathrm{T}_{8}\right)$ data were analyzed by separate 2 (treatment) $\times 2$ (time) repeated-measures analyses of covariance (ANCOVA) for each dependent variable. Differences between treatment groups across time for any variable were determined by significant group $\times$ time interactions. Statistical significance was set at $p<0.05$.

\section{Results}

Table 1 presents the descriptive statistics (mean \pm SD) for the sample. No significant differences were found between the groups for age ( $p=0.34$ ) or body weight ( $p=0.87)$.

Results for the flexibility tests are presented in Table 2. Significant improvements in ROM were made by the AA group for all flexibility measures with the exception of the left side BS and right side SR. In contrast, the control group showed no change in any flexibility measure except for a significant loss in ROM for the right-side KE test. Significant time $\times$ condition interactions were found for all measures $(p<0.01)$. All results favored the AA group.

Results for all functional tests are presented in Table 3. The AA group's CS $(p=0.003)$ and MRPT $(p=0.001)$ performances improved significantly due to training, while $\mathrm{C}$ showed significant declines $(p=0.021$ and $p=0.043$, respectively). Significant time $\times$ condition interactions were also seen for both tests (CS, $p=0.0002$; MRPT, $p=0.0001)$, with the AA group showing significantly better performance across time. The AA group also improved significantly on the AC test $(p=0.0001)$ and GJST ( $p=0.017)$, while $C$ showed no significant change. As with the previous tests, a significant time $\times$ condition interaction was detected for the AC test $(p=0.0001)$ and GJST ( $p=0.006$ ), with the AA group performing significantly better than the $\mathrm{C}$ group.

The AA group significantly reduced the time necessary to complete the UG $(p=0.041)$ and GS $(p=0.041)$ tests, while C showed significant declines in performance (UG, $p=0.007$; $\mathrm{GS}, p=0.043)$. Significant time $\times$ condition interactions were

Table I Participant characteristics (mean \pm SD)

\begin{tabular}{llll}
\hline & Control & Experimental & p value \\
\hline Number $(\mathrm{n})$ & 8 & 9 & \\
Age (years) & $87.5 \pm 6.2$ & $90.0 \pm 4.5$ & 0.34 \\
Weight $(\mathrm{kg})$ & $63.6 \pm 8.3$ & $62.7 \pm 10.3$ & 0.87 \\
\hline
\end{tabular}


Table 2 Pre- and posttest means (standard deviation) for range-of-motion measures of the active-assisted stretching and control groups

\begin{tabular}{|c|c|c|c|c|c|c|c|}
\hline \multirow[t]{2}{*}{ Test } & \multicolumn{3}{|c|}{ Active-assisted stretch } & \multicolumn{3}{|l|}{ Control } & \multirow[b]{2}{*}{$p$ between } \\
\hline & Pretest & Posttest & $p$ within & Pretest & Posttest & $p$ within & \\
\hline Back scratch, right (cm) & $-23.65(20.42)$ & $-24.46(18.39)$ & $0.020 *$ & $-21.03(22.73)$ & $-14.53(21.69)$ & 0.435 & $0.008^{* *}$ \\
\hline Back scratch, left (cm) & $-25.55(12.88)$ & $-27.31(11.96)$ & $0.07 \mid$ & $-25.12(13.72)$ & $-21.44(13.21)$ & 0.147 & $0.020 * *$ \\
\hline Chair sit-and-reach, right $(\mathrm{cm})$ & $-6.07(\mid 4.25)$ & $-1.55(9.17)$ & 0.104 & $-11.76(9.98)$ & $-13.49(10.44)$ & 0.164 & $0.004^{* *}$ \\
\hline Chair sit-and-reach, left $(\mathrm{cm})$ & $-5.08(12.40)$ & $0.43(9.86)$ & $0.035^{*}$ & $-11.76(11.15)$ & $-14.6 \mid(9.40)$ & 0.254 & $0.002^{* *}$ \\
\hline Total body rotation, right $(\mathrm{cm})$ & $12.19(10.51)$ & $24.38(8.15)$ & $0.00 I^{*}$ & $12.45(14.91)$ & II.68 (14.33) & 0.763 & $0.001 * *$ \\
\hline Total body rotation, left $(\mathrm{cm})$ & $15.00(9.80)$ & $23.62(16.15)$ & $0.034 *$ & $15.00(12.14)$ & $11.68(12.10)$ & 0.069 & $0.009 * *$ \\
\hline Knee extension, right (rad) & $2.58(0.19)$ & $2.80(0.13)$ & $0.00 I^{*}$ & $2.50(0.24)$ & $2.4 I(0.2 I)$ & $0.019 *$ & $0.00 I^{* *}$ \\
\hline Knee extension, left (rad) & $2.58(0.19)$ & $2.77(0.13)$ & $0.002^{*}$ & $2.54(0.24)$ & $2.46(0.16)$ & 0.210 & $<0.00 I^{* *}$ \\
\hline
\end{tabular}

Notes: $p$ within, changes between pretest and posttest range-of-motion values for each group. $p$ between, differences between groups across the training period. *Significant change across time. ${ }^{*}$ Significant group $\times$ time interaction.

also seen for both tests (UG, $p=0.041$; GS, $p=0.003$ ), each favoring the AA group.

\section{Discussion}

This is the first study, to our knowledge, to evaluate the impact of AA stretching on ROM and functional performance in a group of older persons residing in a RRC. The fact that increases in ROM were evident across all flexibility tests, with the exception of the left BS and right SR, indicates that the AA stretching program was effective at increasing flexibility in our sample. These results agree with those of other studies reporting significant increases in ROM with PNF stretching programs. ${ }^{7,8}$

The increased performance on the CS test as a result of the AA training program is in agreement with the findings of Swank and colleagues, ${ }^{15}$ who reported that improvements in joint ROM in the elderly were associated with increased ability to rise from a chair. Additionally, the improvement seen in the MRPT with AA training reflects the results of the CS test. Although no studies to date have examined the effect of flexibility training on power output, studies in younger adults support our findings. For example, Hunter and Marshall reported that flexibility training produced significant improvements in countermovement jump height similar to those produced by power training and power and flexibility training combined. ${ }^{16}$ Additionally, Wilson and colleagues showed that eight weeks of static flexibility training, twice a week, enhanced rebound, but not static, bench press performance. ${ }^{17}$

A number of mechanisms may have produced the improvements in power seen with our AA training program. The first is sarcomerogenesis. In their chapter on the morphological bases of muscle power, Edgerton and colleagues noted that increased muscle length should result in increased speed and power production. ${ }^{18}$ Second, increased antagonist compliance may allow more powerful contractions of the agonists. ${ }^{17,19}$ Third, the agonist muscle is used to stretch its antagonist during the active portion of AA stretching, which may have constituted an overload for the agonist and its synergists that increased its contractile strength. Finally, increased elastic compliance of the musculotendinous units may enhance the impact of the stretch-shortening cycle after AA stretching. ${ }^{20}$

Our results differ from those reporting no increases in vertical jump with stretching. ${ }^{21}$ They also appear to conflict with studies that have shown reductions in explosive power as an acute affect of stretching. ${ }^{22-24}$ However, the acute and chronic effects of stretching must be studied in more depth to determine if our results indeed conflict with those examining acute effects or merely constitute an overload and adaptation effect.

The positive effects of AA training on the AC test may seem somewhat peculiar, since no exercise in the training protocol directly targeted the biceps brachii muscles. However, many of the stretching exercises did require active use of the upper body, including the biceps, during the stretch. For example, the back-scratch stretch, targeting the triceps brachii, incorporated the biceps as the active muscle initiating the stretch. The active use of the biceps brachii during this stretch may have served as a training stimulus for that muscle. Also, the increased compliance of the triceps brachii may have decreased both coactivation levels and antagonist "braking" during performance of the AC. Both factors may have increased force production and contraction speed of the biceps, thereby improving AC performance. ${ }^{6}$

The significant improvement we found in GJST performance agrees with the findings of Carmel and colleagues, ${ }^{4}$ who also saw significant increases in this test after AA 
Table 3 Pre- and posttest means (standard deviation) for functional measures of the active-assisted stretching and control groups

\begin{tabular}{|c|c|c|c|c|c|c|c|}
\hline \multirow[t]{2}{*}{ Test } & \multicolumn{3}{|c|}{ Active-assisted stretch } & \multicolumn{3}{|l|}{ Control } & \multirow[b]{2}{*}{$p$ between } \\
\hline & Pretest & Posttest & $p$ within & Pretest & Posttest & $p$ within & \\
\hline Chair stand (repetitions) & II.I (5.2) & $13.0(5.7)$ & $0.003 *$ & $9.88(6.4)$ & $7.75(5.1)$ & $0.021 *$ & $<0.001 * *$ \\
\hline Modified ramp power (watts) & $69.2(20)$ & $86.9(29)$ & $0.001 *$ & $57.0(19)$ & $52.4(18)$ & $0.043^{*}$ & $<0.00 I^{* *}$ \\
\hline Arm curl (repetitions) & $12.9(4.8)$ & $18.8(3.4)$ & $<0.00 I^{*}$ & I5.63 (7.I) & $13.9(5.7)$ & & $<0.00 I^{* *}$ \\
\hline Gallon jug shelf (sec) & $13.4(3.0)$ & II $.5(2.7)$ & $0.017^{*}$ & I8.5 (II) & $18.9(10)$ & & $0.006 * *$ \\
\hline 8' up-and-go (sec) & $8.7(3.1)$ & $7.6(2.4)$ & $0.04 I^{*}$ & $12.0(7.9)$ & I4.6 (II) & $0.007 *$ & $0.003 * *$ \\
\hline Gait speed (sec) & $13.9(3.0)$ & $12.3(3.0)$ & $0.041 *$ & $16.3(6.1)$ & $18.7(3.0)$ & $0.043 *$ & $0.007 * *$ \\
\hline
\end{tabular}

Notes: $p$ within, changes between pre- and posttest functional values for each group. $p$ between, differences between groups across the training period. *Significant change across time. **Significant group $\times$ time interaction.

stretching. One possible mechanism for this improvement was the more effective transfer of force and power from the lower body musculature to the upper body musculature through the core muscles due to improved ROM of the trunk rotators. This factor is especially relevant given the increase in lower and upper body power after our intervention. Another possible contributor to this improvement was the increase in elbow flexor power/endurance as indicated by the significant increase in the number of repetitions performed on the $\mathrm{AC}$ test by the AA group. In addition, the increased ROM of the shoulder extensors, the elbow extensors, and the core muscles may have affected task performance. The shoulder extensors were stretched during the back scratch and back arm press stretches. The core muscles were stretched during the overhead side and seated trunk stretches; and the elbow extensors were stretched during the back scratch stretch. Finally, we acknowledge the contribution of the stretch-shortening cycle to task performance. Because the water-filled jugs used in the assessment weigh about $3.8 \mathrm{~kg}$, the working muscles would have been preloaded before they were used to move each jug. Therefore, the increase in compliance in the muscles of the AA group may have increased stored elastic energy and thus improved test performance.

The positive impact of the AA flexibility program on mobility reflects the link between ROM and functional performance reported by other researchers. ${ }^{4,25,26}$ For example, Gajdosik and colleagues reported faster gait speeds after eight weeks of stretching, and many of the stretches in that study targeted the same joints as the AA stretches in the current study ${ }^{26}$ Carmel and colleagues reported significantly greater increases in gait speed with AA stretching than with isokinetic resistance training at both high and low velocities. ${ }^{4}$ Chan and colleagues also noted better gait economy with increased ROM after flexibility training, suggesting that walking becomes easier as ROM increases. ${ }^{25}$
An indication of the mechanism by which GS was increased in the current study can be drawn from the results reported by DiBenedetto and colleagues, who found that increases in flexibility positively affected stride length. ${ }^{27}$ It is likely that the positive effects on both stride length and gait economy increased maximal walking speed. ${ }^{25}$ The lengthening of the muscles (sarcomerogenesis) due to AA stretching may also have increased their contractile speeds during task performance. ${ }^{18}$

A number of physical factors affect performance on the UG test, including leg strength and power when rising from and sitting down in a chair, walking speed when approaching, rounding, and moving away from the pylon, and dynamic balance or agility in rounding the pylon. Therefore, the improvements in CS and GS discussed earlier would contribute to improved UG performance. Our findings are supported by Gajdosik and colleagues, who noted decreases in the time to complete an agility course and increases in walking speed after an eight-week stretching program. ${ }^{26}$

Our results indicate that eight weeks of AA stretching may be an effective intervention for improving ROM, mobility, and functional power in older persons living in a RRC. AA stretching may be especially effective as an alternative or preliminary training method for frail elders who do not have the physical working capacity to perform more intense interventions such as resistance or cardiovascular training. Our data provide a clear link between flexibility and functional performance in older persons and support the inclusion of flexibility training in interventions designed to increase independence in older persons.

\section{Limitations}

Given the small sample size in this preliminary study, generalizability of the results to the larger population of older persons living in RRCs should be approached with caution. 
Additionally, the accuracy and reliability of these data were limited by a few factors. The first limitation was the ability and willingness of the participants to perform a maximal effort during all testing and training sessions. The second was the potential for interference due to fatigue from previous tests or from other daily activities. Next, the results were limited to the accuracy and reliability of the measuring sticks, hand-held stopwatches, and goniometers and the skill of the tester. These technical issues were addressed in part by all tests being administered by the same tester, who had three years' testing experience with older persons in a RRC environment. Finally, the fact that the tester was not blinded could have potentially biased the results toward the AA group. It should also be remembered that reliability analyses within our laboratory were limited to two flexibility and three functional performance tests.

When working with an older population, it is imperative to create a comfortable relationship between examiners and participants. In order to establish a certain level of familiarity to minimize attrition, the training staff and the testing staff were the same individuals. Although perhaps resulting in increased compliance, this factor prevented the study from being blinded, and the authors acknowledge that as a limitation of these findings.

\section{Acknowledgments}

We would like to thank staff and administration at the Miami Jewish Home and Hospital for their cooperation and provision of space for conducting exercise and functional testing.

\section{Disclosure}

The authors report no conflicts of interest in this work.

\section{References}

1. Allander E, Bjornsson OJ, Olafsson O, Sigfusson N, Thorsteinsson J. Normal range of joint movements in shoulder, hip, wrist and thumb with special reference to side: a comparison between two populations. Int J Epidemiol. 1974;3:253-261.

2. Jette AM, Branch LG, Berlin J. Musculoskeletal impairments and physical disablement among the aged. J Gerontol. 1990;45: M203-M208.

3. Alexander NB, Gross MM, Medell JL, Hofmeyer MR. Effects of functional ability and training on chair-rise biomechanics in older adults. J Gerontol A:Biol Sci Med Sci. 2001;56A:M538-M547.

4. Carmel MP, Czaja S, Asfour S, Khalil T, Morgan RO, Signorile JF. The impact of high-speed versus low-speed isokinetic training on functional performance measures in elderly women. Med Sci Sports Exerc. 2000;32(5 Suppl):S356.

5. Morey MC, Schenkman M, Studenski SA, et al. Spinal-flexibilityplus-aerobic versus aerobic-only training: effects of a randomized clinical trial on function in at-risk older adults. J Gerontol Med Sci. 1999;54A:M335-M342.
6. Burke DG, Pelham TW, Holt LE. The influence of varied resistance and speed of concentric antagonist contractions on subsequent concentric agonist efforts. J Strength Cond Res. 1999;13:193-197.

7. Etnyre BR, Lee EJ. Chronic and acute flexibility of men and women using three different stretching techniques. Res $Q$ Exercise Sport. 1988;59:222-228

8. Sady SP, Wortman M, Blanke D. Flexibility training: ballistic, static or proprioceptive neuromuscular facilitation? Arch Phys Med Rehabil. $1982 ; 63: 261-263$

9. Rikli RE, Jones CJ. Development and validation of a functional fitness test for community-residing older adults. J Aging Phys Activ. 1999;7:129-161.

10. Bandy WD, Irion JM, Briggler M. The effect of time and frequency of static stretching on flexibility of the hamstring muscles. Phys Ther. 1997;77:1090-1096.

11. Hoeger WWK, Hoeger SA. Muscular flexibility assessment and prescription. In: Hoeger WWK, Hoeger SA, editors. Lifetime Physical Fitness and Wellness: A Personalized Program. Englewood, CA: Brooks Cole; 1998. p. 121-140.

12. Signorile JF, Sandler D, Kempner L, Stanziano D, Ma F, Roos BA. The ramp power test: a new method of power assessment for older individuals. J Gerontol Med Sci. 2007;62A:1266-1273.

13. Signorile JF, Sandler D, Ma F, et al. The gallon-jug shelf-transfer test: an instrument to evaluate deteriorating function in older adults. $J$ Aging Phys Activity. 2007;15:56-74.

14. Cress ME, Buchner DM, Questad KA, Esselman PC, deLateur BJ, Schwartz RS. Continuous-scale physical functional performance in healthy older adults: a validation study. Arch Phys Med Rehab. 1996;77:1243-1250.

15. Swank AM, Funk DC, Durham MP, Roberts S. Adding weights to stretching exercise increases passive range of motion for healthy elderly. J Strength Cond Res. 2003;17:374-378.

16. Hunter JP, Marshall RN. Effects of power and flexibility training on vertical jump technique. Med Sci Sport Exerc. 2002;34:478-486.

17. Wilson GJ, Elliott BC, Wood GA. Stretch-shortening cycle performance enhancement through flexibility training. Med Sci Sports Exerc. 1992; 24:116-123.

18. Edgerton VR, Roy RR, Gregor RJ, Rugg S. Morphological basis of skeletal muscle power. In: Jones NL, McCartney N, McComas AJ, editors. Human Muscle Power. Champaign, IL: Human Kinetics; 1986. p. 43-64.

19. Worrell TW, Smith TL, Winegardner J. Effect of hamstring stretching on hamstring muscle performance. J Orthop Sports Phys Ther. 1994;20:154-159.

20. Macaluso A, De Vito G. 2004. Muscle strength, power and adaptations to resistance training in older people. Eur J Appl Physiol. 91:450-472.

21. Knudson D, Bennett K, Corn R, Leick D, Smith C. Acute effects of stretching are not evident in the kinematics of the vertical jump. $J$ Strength Cond Res. 2001;15:98-101.

22. Bergstrom G, Aniansson A, Bjelle A, Grimby G, Lundgren-Lindquist B, Svanborg A. Functional consequences of joint impairment at age 79. Scand J Rehab Med. 1985;17:183-190.

23. Kokkonen J, Nelson AG, Cornwell A. Acute muscle stretching inhibits maximal strength performance. Res Q Exerc Sport. 1998;69: 411-415.

24. Nelson AG, Driscoll NM, Landin DK, Young MA, Schexnayder IC. Acute effects of passive muscle stretching on sprint performance. J Sports Sci. 2005;23:449-454.

25. Chan SP, Hong Y, Robinson PD. Flexibility and passive resistance of the hamstrings of young adults using two different static stretching protocols. Scand J Med Sci Sports. 2001;11:81-86.

26. Gajdosik RL, Vander Linden DW, McNair PJ, Williams AK, Riggin TJ. Effects of an eight-week stretching program on passive-elastic properties and function of the calf muscles of older women. Clin Biomech. 2005;20:973-983.

27. DiBenedetto M, Innes KE, Taylor AG, et al. Effects of a gentle Iyengar yoga program on gait in the elderly: an exploratory study. Arch Phys Med Rehabil. 2005;86:1830-1837. 